\title{
Factores asociados a síntomas depresivos en trabajadoras sexuales
}

\author{
Factors associated to depression symptomatology in sex workers
}

\author{
Ingrid Mercedes Bohórquez ${ }^{1}$, Sandy Caballero ${ }^{1}$, Lourdes Carrera ${ }^{1,2}$, Rosario Chávez ${ }^{1,2}$, \\ Rocío Espinoza ${ }^{1}$, Letsie Flores ${ }^{1}$, Miguel Llanos ${ }^{1}$, Elena Luna ${ }^{1}$, Juan Vega ${ }^{1}$, José Vera ${ }^{1}$, \\ Héctor Salvatierra ${ }^{3}$, Héctor Pereyra ${ }^{3}$ \\ ${ }^{1}$ Estudiante, Escuela Académico Profesional de Medicina Humana, Universidad Nacional Mayor de San Marcos, UNMSM. \\ ${ }^{2}$ Miembro de Grupo de estudio Asociación para el Desarrollo de la Investigación Estudiantil en Ciencias de la Salud, ADIECS, UNMSM. \\ ${ }^{3}$ Médico Asesor.
}

\begin{abstract}
Resumen
Introducción: Las condiciones de trabajo y la violencia ejercida contra las trabajadoras sexuales han sido relacionadas con el incremento de las tasas de infecciones de transmisión sexual. Sin embargo, también se encuentran enfermedades que afectan su salud mental. Objetivos: Determinar la magnitud de síntomas depresivos y factores asociados en trabajadoras sexuales. Diseño: Estudio transversal-analítico. Lugar: Centro de salud referencial para la prevención y control de infecciones de transmisión sexual. Participantes: Trabajadoras sexuales. Intervenciones: A 117 trabajadoras sexuales, que fueron atendidas durante los meses de mayo y junio del 2010, se aplicó el test de Beck para medir la sintomatología depresiva y una encuesta para medir los siguientes factores: socio-demográficos, sociales (antecedentes de maltrato físico, psicológico y sexual en el trabajo y hábitos nocivos: alcohol y drogas), conductas sexuales de riesgo y características del trabajo (edad de inicio, años de trabajo, satisfacción con el trabajo). Principales medidas de resultados: Porcentaje de síntomas depresivos y fuerza de asociación entre los factores y la presencia de síntomas depresivos. Resultados: El 56,4\% de las trabajadoras sexuales presentó algún nivel de sintomatología depresiva. El nivel de síntomas depresivos graves estuvo presente en $23,9 \%$. Los factores que se encontraron asociados a depresión, independiente del nivel, fue el antecedente de maltrato físico frecuente, con $0 R=9,46$, IC95\% 1,05 a 85,07; $p=0,045$. Los factores asociados a nivel de síntomas depresivos graves fueron: ingreso económico menor a S/. 1 000.00, tener hijos, haberse iniciado en el trabajo sexual antes de los 18 años y consumo de drogas $(p<0,05)$. Conclusiones: Más de $50 \%$ de trabajadoras presentó algún nivel de síntomas depresivos, que estuvieron asociados al antecedente de maltrato físico. Para el nivel síntomas depresivos graves, los factores asociados fueron: ingreso económico menor a S/. 1000.00 , tener hijos, haberse iniciado en el trabajo sexual antes de los 18 años y consumir drogas.

Palabras clave: Depresión; prostitución; mujeres maltratadas; violencia.
\end{abstract}

\begin{abstract}
Introduction: The working conditions and violence against sex workers has been linked to increasing rates of sexual transmitted diseases. But there are also other diseases that affect their mental health. Objectives: To determine the magnitude of depressive symptoms and associated factors in sex workers. Design: Crossanalytic study. Setting: Health Centre of reference for the prevention and control of sexually transmitted infections. Participants: Sex workers. Interventions: Beck test was applied to 117 sex workers attended between May and June 2010 in order to measure depressive symptoms as well as a survey to determine socio-demographic and social (physical abuse, psychological and sexual labor and harmful habits: alcohol and drug abuse) factors, risky sexual behavior and job characteristics (age at onset, years of work, job satisfaction). Main outcome measures: Percentage of depressive symptoms and weight of association between factors and presence of depressive symptoms. Results: Some level of depressive symptoms was found in $56,4 \%$ of sex workers. Severe depressive symptoms were present in $23,9 \%$. Main factor associated with depression was background of frequent physical abuse $(O R=9,46, C 195 \%: 1,05$ to 85,07; $p=0,045)$. Factors associated with severe depressive symptoms were income less than $S / .1000 .00$, having children, having started sex work before age 18 , and drug abuse $(p<0,05)$. Conclusions: Over $50 \%$ of sex workers had some level of depressive symptoms generally associated with history of physical abuse. Severe depressive symptoms were associated to income less than S/. 1000.00 , having children, having started sex work before age 18, and drug abuse.
\end{abstract}

Keywords: Depression; prostitution; battered women; violence.

An Fac med. 2010;71 (4):277-82

\section{INTRODUCCIÓN}

La ONUSIDA, en el año 2003, señala que existen 25000 trabajadoras sexuales en el Perú, perteneciendo a 0,3\% de la población femenina entre 15 y 49 años de edad ${ }^{(1)}$. Las trabajadoras sexuales son afectadas constantemente por las infecciones de transmisión sexual (ITS), incluyendo el virus de inmunodeficiencia humana (VIH). Otros aspectos que ponen en riesgo su vida son la violencia y las condiciones del trabajo a las que se ven sometidas, colocándolas en un grupo vulnerable para enfermedades que afectan su salud mental ${ }^{(2)}$. Algunos estudios han demostrado que esta población, al estar expuesta a mayores factores de riesgo -como violencia y estigmatización-, presenta mayores índices de depresión en comparación con la población en general. En Porto Alegre, Brasil, la prevalencia de depresión en trabajadoras fue de $67 \%$, a diferencia de $14,5 \%$ de depresión en la población femenina de esa ciudad ${ }^{(3)}$.
Este estudio tiene como objetivo determinar la presencia de síntomas depresivos y factores asociados en las trabajadoras sexuales que acuden a un centro de salud referencial para la prevención de ITS y VIH.

\section{MÉTODOS}

El presente es un estudio transversal y analítico. Se realizó un muestreo no probabilístico de tipo casos consecutivos 
en las trabajadoras sexuales, durante los meses de mayo y junio de 2010. Se incluyó las trabajadoras sexuales mayores de edad y que fueron atendidas en el centro de salud durante este periodo. Se excluyó toda encuesta respondida con alguna pregunta incompleta y a las trabajadoras sexuales transexuales.

A las trabajadoras sexuales se les entregó un consentimiento informado, en el que en forma verbal y escrita se explicó los objetivos del estudio, el anonimato de la procedencia de la información brindada y el agradecimiento por su participación voluntaria. Luego de haber sido aceptado el consentimiento informado, se procedió a la administración de la encuesta, que fue conducida en ambientes privados y donde solo el entrevistador estuvo presente durante la recolección de datos.

Se utilizó como instrumento de análisis una encuesta de 43 preguntas divididas en dos secciones. Las primeras 22 evaluaron factores sociodemográficos (edad, lugar de procedencia, lugar de nacimiento, nivel de instrucción, estado civil, ingreso económico mensual, número de hijos, personas con las que vive, antecedente de aborto), factores sociales (consumo de alcohol, drogas, antecedente de maltrato físico y psicológico, coerción sexual), conducta de riesgo sexual (antecedente de infección de transmisión sexual, infección por VIH y uso de preservativo), características del trabajo (edad de inicio, cantidad de años trabajando, conocimiento de los familiares). La segunda sección estuvo constituida por el inventario de depresión de Beck (IDB), instrumento autoadministrado que mide el nivel de síntomas depresivos en los últimos 6 meses. La puntuación del IDB es de 0 a 63. Consideramos como puntos de corte para clasificar los grados de depresión, como sigue: normal (0 a 9), leve (10 a 18 ), moderada (19 a 29) y grave (30 a 63 ). Se consideró como ausencia de síntomas depresivos un puntaje menor a 10 en el $\mathrm{IDB}^{(4)}$. El IDB fue validado en el Perú por el Instituto Nacional de Salud Mental Honorio Delgado - Hideyo Noguchi. El protocolo del estudio fue aprobado por la DIRESA I, Callao- Perú.

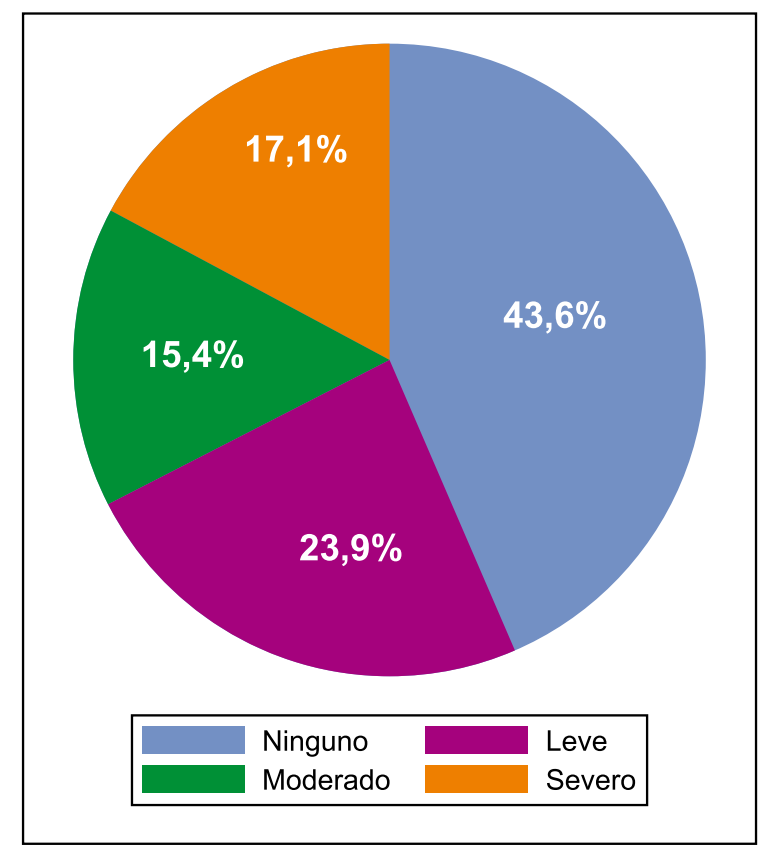

Figura 1. Nivel de síntomas depresivos en trabajadoras sexuales.

Las encuestas desarrolladas por los participantes fueron digitadas en una base de datos, en el programa STATA versión 10. En el análisis descriptivo se determinó la frecuencia de sintomatología depresiva y factores medidos. En el análisis bivariado, se asoció la presencia de síntomas depresivos con cada variable por la prueba chi cuadrado y prueba exacta de Fisher, cuando la frecuencia esperada fue menor a 5 (nivel de confiabilidad al 95\%). Se empleó el análisis de regresión logística, considerando como variable dependiente en el primer caso la presencia de síntomas depresivos y, en el segundo, el nivel de síntomas depresivos.

\section{RESULTADOS}

Se entrevistó a 117 trabajadoras sexuales. La prevalencia de síntomas depresivos fue de $56,4 \%$. Se detalla los niveles de depresión en la figura 1.

El 48,7\% de trabajadoras sexuales entrevistada tenía entre 25 y 34 años de edad. La edad promedio fue 30,1 +/-8,1 años. La edad mínima fue 18 años y la máxima 55. El 33,3\% residía en el cono norte y $55,6 \%$ procedía de ciudades de la región costa. El 56,3\% alcanzó nivel de estudio de secundaria completa y $23,1 \%$ educación superior. El 63,3\% era soltera y $41,9 \%$ vivía con sus hijos y familiares; $57,3 \%$ tenía 1 a 3 hijos y $27,4 \%$ no tenía hijos. El 55,6\% tenía un ingreso económico mayor a S/. 1000.00 soles mensuales. Casi 65\% tenía antecedente de haberse realizado un aborto (tabla 1 ).

El 8,6\% había consumido algún tipo de droga en los últimos 6 meses. Respecto al antecedente de violencia en el trabajo, al menos una vez, 45,3\% refirió haber sido víctima de maltrato psicológico, 17,1\% de maltrato físico y $12,8 \%$ de maltrato sexual (tabla 2).

Respecto a las conductas de riesgo de enfermedades sexuales, 32,5\% comunicó haber recibido tratamiento para alguna infección de transmisión sexual en los últimos 6 meses. Ninguna había sido diagnosticada de VIH hasta el momento que fue entrevistada. Sobre el uso de preservativo, 95,7\% siempre lo empleaba con el cliente y 43,6\% nunca lo empleaba con su pareja.

La edad promedio de inicio como trabajadora sexual fue 25,2 +/- 6,4 años. La edad mínima fue 16 años y la máxima 49. El 58,1\% tenía entre 1 y 5 años laborando como trabajadora sexual. Más de $60 \%$ no se encontraba satisfecha con su 
Tabla 1. Factores socio-demográficos según presencia de síntomas depresivos.

\begin{tabular}{|c|c|c|c|c|}
\hline & \multicolumn{4}{|c|}{ Síntomas depresivos } \\
\hline & \multicolumn{2}{|c|}{ Ausencia } & \multicolumn{2}{|c|}{ Presencia } \\
\hline & $\mathrm{N}$ & $\%$ & $\mathrm{~N}$ & $\%$ \\
\hline \multicolumn{5}{|l|}{ Edad (años) } \\
\hline $18-24$ & 12 & 23,5 & 17 & 25,8 \\
\hline $25-34$ & 25 & 49,0 & 32 & 48,5 \\
\hline $35-44$ & 10 & 19,6 & 13 & 19,7 \\
\hline$>45$ & 4 & 7,8 & 4 & 6,1 \\
\hline \multicolumn{5}{|l|}{ Residencia actual } \\
\hline Cono norte & 16 & 31,4 & 23 & 34,9 \\
\hline Cono sur & 8 & 15,7 & 6 & 9,1 \\
\hline Cono este & 9 & 17,7 & 11 & 16,7 \\
\hline Cono centro & 9 & 17,7 & 10 & 15,2 \\
\hline Callao & 9 & 17,7 & 16 & 24,2 \\
\hline \multicolumn{5}{|c|}{ Lugar de nacimiento } \\
\hline Costa & 26 & 50,9 & 39 & 59,1 \\
\hline Sierra & 14 & 27,5 & 15 & 22,7 \\
\hline Selva & 11 & 21,6 & 12 & 18,2 \\
\hline \multicolumn{5}{|l|}{ Nivel de estudios } \\
\hline Primaria & 3 & 5,9 & 12 & 18,2 \\
\hline Secundaria & 30 & 58,8 & 37 & 56,1 \\
\hline Técnica & 14 & 27,5 & 13 & 19,7 \\
\hline Universitaria & 4 & 7,8 & 4 & 6,1 \\
\hline \multicolumn{5}{|l|}{ Estado civil } \\
\hline Soltera & 35 & 68,6 & 39 & 59,1 \\
\hline Casada & 5 & 9,8 & 3 & 4,6 \\
\hline Conviviente & 8 & 15,7 & 23 & 34,9 \\
\hline Divorciada & 3 & 5,9 & 1 & 1,5 \\
\hline \multicolumn{5}{|c|}{ Personas con quien vive } \\
\hline Sola & 5 & 9,8 & 15 & 22,7 \\
\hline Con su pareja & 6 & 11,8 & 9 & 13,6 \\
\hline Con hijos & 17 & 33,3 & 14 & 21,2 \\
\hline Con familiares & 22 & 43,1 & 27 & 40,9 \\
\hline Amigos u otros & 1 & 1,9 & 1 & 1,5 \\
\hline \multicolumn{5}{|c|}{$\begin{array}{l}\text { Ingreso económico } \\
\text { (nuevos soles) }\end{array}$} \\
\hline $200-399$ & 3 & 5,9 & 7 & 10,6 \\
\hline $400-599$ & 3 & 5,9 & 3 & 4,6 \\
\hline $600-799$ & 5 & 9,8 & 13 & 19,7 \\
\hline $800-999$ & 10 & 19,6 & 8 & 12,1 \\
\hline$>1000$ & 30 & 58,8 & 35 & 53,0 \\
\hline \multicolumn{5}{|c|}{ Número de hijos vivos } \\
\hline 0 & 16 & 31,4 & 16 & 24,2 \\
\hline $1-3$ & 25 & 49,0 & 42 & 63,6 \\
\hline$>3$ & 10 & 19,6 & 8 & 12,1 \\
\hline \multicolumn{5}{|l|}{ Número de abortos } \\
\hline 0 & 15 & 30,8 & 21 & 31,8 \\
\hline $1-3$ & 36 & 63,3 & 38 & 57,6 \\
\hline$>3$ & 0 & 0 & 7 & 10,6 \\
\hline
\end{tabular}

trabajo y $94,9 \%$ de los familiares de las trabajadoras sexuales desconocían de su labor (tabla 3).

La prueba chi cuadrado mostró asociación de la presencia de síntomas depresivos con el número de abortos $(\mathrm{p}=0,037)$, el consumo de drogas $(p=0,005)$ y el conocimiento del trabajo que realizaban, por sus familiares $(p=0,025)$. Mediante el análisis de regresión logística, ajustado para la presencia de síntomas depresivos, se encontró asociación con el antecedente de violencia física frecuente $(\mathrm{p}=0,045)$.

Los resultados de la regresión logística teniendo como variable dependiente el nivel de síntomas depresivos mostró lo siguiente: para las personas que presentaron síntomas depresivos leves, se encontró asociación con el nivel de ingreso económico menor a mil soles; en las personas que presentaron nivel de síntomas depresivos moderados, se halló asociación con el antecedente de un número de abortos mayor a 3; y en las personas que presentaron un nivel de síntomas depresivos graves, se encontró asociación con la ausencia y frecuencia regular de antecedentes de violencia psicológica, la edad de inicio mayor a los 18 años, el número de hijos mayor a 1 y el ingreso económico menor a mil soles. Los resultados son descritos con mayor detalle en la tabla 4.

\section{DISCUSIÓN}

Encontramos que más de 50\% de la muestra estudiada presentaba algún nivel de síntoma depresivo. En otros estudios en trabajadoras sexuales -como el realizado en Porto Alegre (Brasil), en una muestra de 97 trabajadoras sexuales-, la prevalencia de síntomas depresivos fue $67 \%$, asociada al consumo de alcohol y antecedentes de ITS ${ }^{(3)}$. En Puerto Rico, en una muestra de 127 trabajadoras sexuales fue $70 \%$, en el que se demostró que las mujeres con depresión no utilizaban el preservativo de manera constante y consistente $^{(5)}$. En Guanxi (China) hallaron una prevalencia de síntomas depresivos de 62\% (2006) en 300 trabajadoras sexuales y $30 \%$ (2009) en una muestra de 122 trabajadoras sexuales ${ }^{(6,7)}$. En ex 
Tabla 2. Factores sociales según presencia de síntomas depresivos.

\begin{tabular}{|c|c|c|c|c|}
\hline & \multicolumn{4}{|c|}{ Síntomas depresivos } \\
\hline & \multicolumn{2}{|c|}{ Ausencia } & \multicolumn{2}{|c|}{ Presencia } \\
\hline & $\mathrm{N}$ & $\%$ & N & $\%$ \\
\hline \multicolumn{5}{|l|}{$\begin{array}{l}\text { Frecuencia de } \\
\text { consumo de alcohol }\end{array}$} \\
\hline No consume & 16 & 31,4 & 19 & 28,8 \\
\hline 1 vez al mes & 17 & 33,3 & 25 & 37,9 \\
\hline $2-3$ veces al mes & 2 & 3,9 & 3 & 4,6 \\
\hline 1 vez por semana & 11 & 21,6 & 15 & 22,7 \\
\hline$>1$ vez por semana & 5 & 9,8 & 4 & 6,1 \\
\hline \multicolumn{5}{|l|}{ Consumo de drogas } \\
\hline No & 51 & 100 & 56 & 84.9 \\
\hline Sí & 0 & 0 & 10 & 15,2 \\
\hline \multicolumn{5}{|c|}{ Violencia psicológica } \\
\hline Nunca & 32 & 62,8 & 32 & 48,5 \\
\hline Casi nunca & 6 & 11,8 & 8 & 12,1 \\
\hline Algunas veces & 12 & 23,5 & 21 & 31,8 \\
\hline Bastantes veces & 1 & 2,0 & 3 & 4,6 \\
\hline \multicolumn{5}{|l|}{ Violencia física } \\
\hline Nunca & 47 & 92,2 & 50 & 75,8 \\
\hline Casi nunca & 3 & 5,9 & 9 & 13,6 \\
\hline Casi siempre & 1 & 2,0 & 5 & 7,6 \\
\hline A veces & & & 1 & 1,5 \\
\hline \multicolumn{5}{|l|}{ Violencia sexual } \\
\hline Nunca & 46 & 90,2 & 56 & 84,9 \\
\hline Casi nunca & 2 & 3,9 & 2 & 3,0 \\
\hline Algunas veces & 2 & 3,9 & 7 & 10,6 \\
\hline Bastantes veces & 1 & 2,0 & 1 & 1,5 \\
\hline
\end{tabular}

Tabla 3. Características del trabajo según presencia de síntomas depresivos.

\begin{tabular}{|c|c|c|c|c|}
\hline & \multicolumn{4}{|c|}{ Síntomas depresivos } \\
\hline & \multicolumn{2}{|c|}{ Ausencia } & \multicolumn{2}{|c|}{ Presencia } \\
\hline & $\mathrm{N}$ & $\%$ & $\mathrm{~N}$ & $\%$ \\
\hline \multicolumn{5}{|l|}{ Edad de inicio } \\
\hline$<18$ & 3 & 5,9 & 5 & 7,6 \\
\hline $18-25$ & 23 & 45,1 & 34 & 51,5 \\
\hline $26-35$ & 20 & 39,2 & 22 & 33,3 \\
\hline$>35$ & 5 & 9,8 & 5 & 7,6 \\
\hline \multicolumn{5}{|l|}{ Años trabajando } \\
\hline$<1$ año & 8 & 15,7 & 16 & 24,2 \\
\hline 1 - 5 años & 31 & 60,8 & 37 & 56,1 \\
\hline$>5$ años & 12 & 23,5 & 13 & 19,7 \\
\hline \multicolumn{5}{|c|}{ Satisfacción con el trabajo } \\
\hline Muy satisfecha & 0 & 0 & 1 & 1,5 \\
\hline Satisfecha & 8 & 15,7 & 6 & 9,1 \\
\hline Indiferente & 15 & 29,4 & 13 & 19,7 \\
\hline No satisfecha & 22 & 43,1 & 33 & 50,0 \\
\hline Muy insatisfecha & 6 & 11,8 & 13 & 19,7 \\
\hline \multicolumn{5}{|c|}{$\begin{array}{c}\text { Conocimiento de sus familiares } \\
\text { del trabajo que realiza }\end{array}$} \\
\hline No & 51 & 100 & 60 & 90,9 \\
\hline Sí & 0 & 0 & 6 & 9,1 \\
\hline
\end{tabular}

trabajadoras sexuales, el porcentaje de la población afectada por la depresión ha sido mayor que en las trabajadoras sexuales activas, habiéndosela relacionado con la presencia de otros trastornos mentales, como ansiedad y estrés postraumático ${ }^{(8)}$. En ellas se ha mostrado, además, como un problema incapacitante, acompañado de psicosis y desórdenes alimenticios ${ }^{(9)}$. La presencia de depresión ha sido descrita también como una pérdida del autocontrol de problemas personales y la toma de decisiones, y contribuyen a un mayor grado de aislamiento y menos accesibilidad para los esfuerzos de prevención ${ }^{(10)}$.

La depresión mayor -que en este estudio se manifestó en $17,1 \%$ - es causa de intentos de suicidio, constituyendo el predictor más fuerte para ideación suicida, pero menos poderoso en predecir la progresión de la ideación suicida a la conducta suicida ${ }^{(11)}$.

Todas las formas de violencia han sido vinculadas a niveles altos de depresión. El problema de violencia de género constituye un problema de salud global y derechos humanos que ha conducido a tasas altas de mortalidad y morbilidad, incluyendo problemas de ITS, dependencia de sustancias ${ }^{(12)}$. En el Perú, el estudio de diagnóstico de violencia y trabajo sexual $^{(2)}$ describe que las trabajadoras sexuales son víctimas de violencia por su trabajo (cliente, proxeneta), pareja y entorno social. Los actos de violencia más frecuentes ejercidos contra ella en el trabajo sexual fueron desde agresión física, psicológica, sexual, robo, humillación, despojo de sus ropas, detención arbitraria y abandono en lugares alejados. En Tailandia, el porcentaje de trabajadoras sexuales que mencionó haber sido víctima de algún tipo de violencia fue $14,6 \%{ }^{(13)}$. En China, las mujeres que habían sido víctimas de coerción sexual habían comunicado consumo de alcohol antes de tener sexo con el cliente, y un tercio reportó antecedente de ITS ${ }^{(7)}$. Otro factor que es considerado como un tipo violencia psicológica es la estigmatización, propia de la naturaleza del trabajo que realizan. El estrés y estigma producto de las relaciones interpersonales por el trabajo que realizan han sido descritas como factores que disminuyen 
Tabla 4. Regresión logística según el nivel de síntomas depresivos.

\begin{tabular}{lcccc}
\hline Síntoma depresivo & Variable & OR & Intervalo confianza 95\% & $p$ \\
\hline Leve & Ingreso económico & & & \\
& $600-800$ & 0,04 & $0,0-0,9$ & 0,04 \\
& $800-1000$ & 0,02 & $0,0-0,4$ & 0,01 \\
Moderado & $>1000$ & 0,24 & $0,0-2,1$ & 0,20 \\
& Abortos & & & \\
Grave & $1-3$ & 1,7 & $0,4-7,4$ & 0,51 \\
& $>3$ & 72,6 & $1,9-2800,7$ & 0,02 \\
& Maltrato físico & & & \\
& Nunca & 0,01 & $0,0-0,1$ & 0,001 \\
& Edad de inicio & & & \\
$<18$ & 5,9 & $0.0-0.2$ & 0,02 \\
& $26-35$ & 3,1 & $0,0-0,1$ & 0,04 \\
& Número de hijos & & & \\
$1-3$ & 1,7 & $9,2-32,7$ & 0,02 \\
& $>3$ & 2,7 & $49,1-150,0$ & 0,01 \\
& Ingreso económico & & & \\
$400-600$ & 2,8 & $0,01-0,05$ & 0.02 \\
& $800-1000$ & 9,6 & $-0,01-0,05$ & 0,01 \\
& Consumo de drogas & 6,0 & $1,4-25,9$ & 0,02 \\
\hline
\end{tabular}

el apoyo social a este grupo ${ }^{(14)}$. Además de su relación con la presencia de síntomas depresivos, en otros estudios, la violencia ha sido asociada con el estrés postraumático $^{(7)}$.

En México, en un grupo de trabajadoras sexuales producto del tráfico de humanos, el $65 \%$ de una muestra de 60 había tenido un aborto, y más de la mitad no había sido realizado en un centro de salud, sino fue producto de la violencia ejercida contra ellas ${ }^{(15)}$. En otro estudio, la tasa de abortos espontáneos estuvo asociada con violencia, y la tasa de abortos inducidos, por la coacción de sus parejas, proxenetas y encargados de su centro de trabajo ${ }^{(16)}$. La presencia de un número de abortos mayor a 2 indica la falta inadecuada de métodos anticonceptivos, así como a un mayor riesgo de complicaciones maternas y del feto. Otras mujeres deciden abortar, debido a que la posibilidad de un embarazo 'pone en peligro su actividad' ${ }^{(17)}$.

Los ingresos económicos en este trabajo estuvieron asociados a un nivel moderado y severo de depresión. Se ha descrito que el motivo más frecuente para entrar al mundo del trabajo sexual es este factor, que en la mayoría de casos obedece a causas de necesidad, obligaciones con la familia, pago de deudas, mejorar su situación financiera, haber sido víctima de un fraude y migración ${ }^{(18,19)}$. Por otro lado, muchas mujeres son las responsables económicas absolutas de su familia. En la India, el promedio de las ganancias de las trabajadoras sexuales por cliente es 15 dólares: más de la tercera parte entrega sus ganancias a otras personas, incluyendo proxenetas, policías, entre otros ${ }^{(20,21)}$.

El número de hijos mayor a uno refiere mayor responsabilidad económica y ha sido relacionado con el aumento de riesgo de ITS ${ }^{(22)}$ e intentos de suicidio ${ }^{(23)}$.

Es necesario incorporar servicios de salud mental para las personas que ejercen el trabajo sexual, a fin de que puedan sobreponerse a la violencia que viven diariamente y a sus consecuencias (consumo de drogas, alcohol, violencia interiorizada, estigma interno), por lo que se propone intervenciones para mejorar la salud mental y fuentes de apoyo social para este grupo; de esta forma se brindará ánimo y soporte a las personas que deseen dejar el trabajo. Se sugiere que las intervenciones dirigidas a la prevención de ITS/VIH en este grupo deban estar orientadas en pro de su salud mental. En la ciudad de Londres, los resultados de la instalación de un servicio de atención psicológica han sido hasta el momento buenos y que demuestran que la prevalencia de ITS y conductas sexuales de riesgo han disminuido en el grupo de trabadoras sexuales en $5 \%$ por un año ${ }^{(24)}$.

Las limitaciones de este estudio es el diseño transversal y la aplicación de la técnica de muestreo de tipo no probabilístico. Por este motivo, necesitamos ser cuidadosos al momento de interpretar y generalizar los resultados. Además, se sugiere ampliar el estudio con trabajadoras sexuales en sus propios centros de trabajo. Considerar un estudio de factores determinantes de violencias y las personas por las que son ejercidas.

\section{REFERENCIAS BIBLIOGRÁFICAS}

1. ONUSIDA. Trabajo Sexual y VIH/SIDA. Colección prácticas óptimas del ONUSIDA. 2003. Disponible en: http://data.unaids.org/Publications/IRCpub02/JC705-SexWork-TU_es.pdf. [Consulta: 3 abril de 2010].

2. Salazar $X$. Informe final diagnóstico de la violencia contra los y las trabajadores/as sexuales, mujeres, transgéneros y varones y su vulnerabilidad. MINSA, Perú, julio 2009.

3. Schreiner L, Paim LL, Ramos F, Cunha E, Martins D, Silva J, Lopes C, et al. Prevalência de sintomas depressivos em uma amostra de prostitutas de Porto Alegre. Rev psiquiatr. 2004;26(1):13-20.

4. Rudd MD, Rajab M. Specificity of the Beck Depression Inventory and the confounding role of comorbid disorders in a clinical sample. Cogn Ther Res. 1995;19:51-68.

5. Alegria M, Vera M, Freeman DH, Robles R, Santos MC, Rivera CL. HIV infection, risk behaviors, and depressive symptoms among Puerto Rican sex workers. Am J Public Health. 1994;84:2000-2.

6. Hong Y, Li X, Fang X, Zhao R. Depressive symptoms and condom use with clients among female sex workers in China. Sex Health. 2007;4(2):99104.

7. Hong Y, Fang X, Li X, Liu Y, Li M, Tai-Seale T. Selfperceived stigma, depressive symptoms, and suicidal behaviors among female sex workers in China. J Transcult Nurs. 2010;21(1):29-34. 
8. Ward H, Day S. What happens to women who sell sex? Report of a unique occupational cohort. Sex Transm Infect. 2006;82:413-7.

9. Yung TE, Song JM, Chong J, Seo HJ, Chae JH. Symptoms of posttraumatic stress disorder and mental health in women who escaped prostitution and helping activists in shelters. Yonsei Med J. 2008;49(3):372-82.

10. Romans SE, Potter K, Martin J, Herbison P. The mental and physical health of female sex workers: a comparative study. Aust N Z J Psychiatry. 2001;35:75-80.

11. Nock MK, Hwang I, Sampson N, Kessler RC, Angermeyer M, Beautrais A, et al. Cross-national analysis of the associations among mental disorders and suicidal behavior: findings from the WHO World Mental Health Surveys. Mental Disorders and Suicidal Behavior. PloS Med. 2009;6(8):1-17.

12. Shahmanesh M, Wayal S, Cowan F. Suicidal behavior among female sex workers in Goa, India: the silent epidemic. Am J Public Health. 2009;99(6):1239-46.

13. Decker MR, McCauley HL, Phuengsamran D, Janyam S, Seage GR, Silverman JG. Violence victimisation, sexual risk and sexually transmitted infection symptoms among female sex workers in Thailand. Sex Transm Infect. 2010;14:1-5.

14. Ulibarri M, Semple SJ, Rao S, Strathdee SA, FragaVallejo MA, Bucardo J, et al. History of abuse and psychological distress symptoms among female sex workers in two Mexico-U.S. border cities. Violence Vict. 2009;24(3):399-413.
15. Acharya AK. Sexual violence and proximate risks: a study on trafficked women in Mexico City. Gender, Technology and Development. 2008;12(1):77-99.

16. Shannon K, Kerr T, Strathdee S A, Shoveller J, Montaner J S, Tyndall M W. Prevalence and structural correlates of gender based violence among a prospective cohort of female sex workers. Brit Med J. 2009;339:2939-42.

17. Dalla RL, Xia Y, Kennedy H. "You just give them what they want and pray they don't kill you". Street-Level Sex Workers' Reports of Victimization, Personal Resources, and Coping Strategies. Violence Against Women. 2003;9(11):1367-94.

18. Lafaurie M, Gómez-Sánchez P, Bernal A, Gualteros M, Hassan Vi, Lozano J y col. Embarazo en mujeres en situación de prostitución: Autocuidado y prácticas de riesgo. Rev Colomb Obstet Ginecol. 2008;59(4):285-96.

19. Priscilla M, Pyett R, Benjamin R. Snow JD. Who works in the sex industry? A profile of female prostitutes in Victoria. Aust N Z J Public Health. 1996;20:431-3.

20. Bowen KJ, Dzuvichu B, Rungsung R, Devine AE, Hocking J, Kermode M. Life circumstances of women entering sex work in Nagaland, India. AsiaPacific J Publ Health. 2010;20(2):1-9.

21. Shannon K, Kerr T, Strathdee, S, Shoveller J, Montaner JS, Tyndall M. Prevalence and structural correlates of gender based violence among a prospective cohort of female sex workers. Brit Med J. 2009;339:2939-46.
22. Decker MR, McCauley HL, Phuengsamran D. Violence victimization, sexual risk and sexually transmitted infection symptoms among female sex workers in Thailand. Sex Transm Infect. 2010;86(3):236-40.

23. Hong Y, Li X. Correlates of suicidal ideation and attempt among female sex workers in China. Health Care Women Int. 2007;28(5):490-505.

24. Stevenson C, Petrak J. Setting up a clinical psychology service for commercial sex workers. Int J STD AIDS. 2007;18:231-4.

Financiamiento: Investigación autofinanciada

Conflictos de intereses: Los autores declaran ningún conflicto de interés.

Trabajo ganador del Premio de la sección Alumnos de las IX Jornadas Científicas Sanfernandinas, XII Jornadas de Investigación en Salud, XIX Jornadas Sanfernandinas Estudiantiles, de la Facultad de Medicina, UNMSM, y Premio al Mejor Trabajo de Alumnos de Medicina, de la Asociación de Exalumnos Sanfernandinos de la Peruvian American Medical Society-PAMS, setiembre 2010.
Correspondencia:
Lourdes del Rocío Carrera Acosta
lulurcarrera@yahoo.com 